\title{
DETERMINANTES DE LA EFICIENCIA TECNICA DE EXPLOTACIONES DE FRIJOL UBICADAS EN PORTUGUESA, VENEZUELA
}

\section{DETERMINANTS OF TECHNICAL EFFICIENCY BEAN FARM LOCATED IN PORTUGUESE, VENEZUELA}

\author{
Trifina E. Márquez ${ }^{1}$, Adelis R. Velásquez ${ }^{2}$, José O. Flores ${ }^{3 *}$, Sandra L. Flores ${ }^{4}$, Hernando J. \\ Garzón ${ }^{5}$
}

Recibido para publicación: Junio 19 de 2013 - Aceptado para publicación: Diciembre 03 de 2013

\begin{abstract}
RESUMEN
Se evaluó la eficiencia técnica de explotaciones agrarias con producción de frijol ubicadas en el Sector La Palaciera, municipio San Genaro de Boconoíto del estado Portuguesa, Venezuela. De la muestra de 30 fincas se recabó, mediante cuestionarios, información de cinco insumos y un producto, que corresponden al cultivo sembrado en el año 2010, así como de variables de tipo socioeconómico y geoespacial. Se empleó el método Análisis Envolvente de Datos (DEA, por sus siglas en inglés), con un modelo orientado al producto. Según los resultados, en promedio, la eficiencia técnica global (ETG) fue de 81,2\%, desglosada en una eficiencia técnica pura (ETP) de 86,0\% y una eficiencia de escala (EE) de 95,1\%. Las ineficiencias causadas por la tecnología son mayores que las generadas por un tamaño o escala de producción subóptimo. Según las metas del plan formulado para la muestra de fincas, se puede elevar la producción agregada de frijol de 234.970 $\mathrm{kg}$ a $289.200 \mathrm{~kg}$, lo que supone un incremento de $54.230 \mathrm{~kg}(23,1 \%)$, sin aumentar la cantidad de insumos aplicados actualmente. Las variables socioeconómicas y las de tipo geoespacial no determinaron los niveles de eficiencia técnica de las fincas.
\end{abstract}

Palabras Clave: eficiencia, DEA, insumo, producto, sig, Tobit

\begin{abstract}
It was evaluated the technical efficiency of farms with bean production located in The Palaciera Sector, municipality of San Genaro Boconoito, Portuguesa State, Venezuela. From the sample of 30 farms was collected through questionnaires, information from five inputs and one product, corresponding to the crop planted in

\footnotetext{
Ejercicio profesional. Barinas 5201, Venezuela. Email: elizamarquez2010@hotmail.com

${ }^{2}$ Fondo para el Desarrollo Agrario Socialista (Fondas). Guanare 3350, Estado Portuguesa, Venezuela. Email: adelisvelasquez8@gmail.com ${ }^{3}$ UNELLEZ-Guanare. Antiguo Convento de San Francisco, carrera 3 entre carrera 16 y 17, Mesa de Cavaca 3350, Guanare, Estado Portuguesa, Venezuela. Email: joseovidioflores@gmail.com.

${ }^{4}$ Depto. Ingeniería de Organización, Administración de Empresa y Estadística, Universidad Politécnica de Madrid. C/José Gutierrez Abascal, 2.28006 Madrid, España. Email: sandralizbethflores@gmail.com
}

${ }^{5} U N E F A$. Vía El Toreño, 5201 Barinas, Venezuela. Email: hernandojosegarzon@gmail.com
\end{abstract}


2010, as well as socio-economic and geospatial variables. Data Envelopment Analysis (DEA) method was used with a product-oriented model. According to the results, on average, the overall technical efficiency (GTE) was $81.2 \%$, disaggregated into pure technical efficiency (PTE) of $86.0 \%$ and a scale efficiency (SE) of $95.1 \%$. The inefficiencies caused by the technology are higher than those generated by a sice or scale of sub optimal production. According to the plan's goals formulated for the sample of farm, aggregated production of beans can increase from $234.970 \mathrm{~kg}$ to $289.200 \mathrm{~kg}$, representing an increase of $54.230 \mathrm{~kg}(23.1 \%)$ without increasing the amount of input current applied. The socioeconomic variables and those of geospatial type not determined the levels of technical efficiency of the farms.

Key words: efficiency, DEA, input, output, Gis, Tobit

\section{INTRODUCCIÓN}

El frijol Vigna unguiculata (L.) Walp. es una leguminosa de grano cultivada desde épocas remotas con fines alimenticios en el trópico occidental africano y en varios países americanos. En Venezuela, el frijol suele ser un cultivo de subsistencia para la población campesina, pero a consecuencia de la crisis económica que vive el país, su demanda se ha visto incrementada debido a que constituye una fuente barata de proteína para los estratos sociales de menores ingresos que viven en las zonas marginales de las grandes ciudades, razón por la cual se requiere incrementar su rendimiento y superficie de siembra (Piccirillo e Higuera 1997), a fin de superar la condición deficitaria medida por la demanda aparente de este grano (FUNICA 2011).

Según Flores (2005) el frijol común es una leguminosa alimenticia de gran valor nutritivo y comercial en Venezuela, ocupando el segundo lugar de producción después de la caraota (Phaseolus vulgaris L.). El frijol común y otras especies de Vigna como el frijol mungo y el bucarito, por su adaptación a condiciones de clima seco, presentan ventajas comparativas con relación a otros cultivos, cuando se siembran en la región de los llanos venezolanos. No obstante, son cultivos considerados de baja producción. Para el año 2002 se obtuvieron 13.338 toneladas de frijoles, con un rendimiento promedio nacional bajo de $750 \mathrm{~kg} / \mathrm{ha}$, debido principalmente a las políticas agrícolas existentes en el país, el mal manejo del cultivo y la falta de apoyo en la investigación, lo cual limitó su desarrollo (Marín 2002). Para el año 2011 se estimó una producción de 48.270 toneladas obtenidas en 40.225 ha, para un promedio de $1.200 \mathrm{~kg} / \mathrm{ha}$ de frijol (Fedeagro 2012).

Además de los riesgos inherentes a la actividad, las explotaciones de frijol confrontan otros tipos de problemas que inciden directamente en su eficiencia. Por una parte, los costos de producción se han incrementado de manera significativa en los últimos años y, por la otra, desde el año 2005 la población ocupada en actividades agrícolas tiende a disminuir en términos absolutos, representando en el 2008 un 11\% menos que la del 2005 (Hernández 2009), lo cual presagia mayores dificultades para contratar mano de obra. Asimismo, los precios del frijol pueden tender hacia el estancamiento, como un efecto indirecto de la nueva legislación nacional en materia de regulación de precios de bienes y servicios, lo que implicaría rezagos de los incrementos de los precios con respecto a los costos de los insumos que derivarían, a su vez, en ganancias decrecientes para los agricultores. En este contexto, la solución más viable para 
los agricultores consiste en producir con la mayor eficiencia factible, elevando los niveles de producción y en la medida de lo posible, no aumentar las cantidades de insumos que se están aplicando en la actualidad.

Diversos autores han evaluado el uso de los recursos en explotaciones agrícolas en Venezuela, desde el punto de vista de la productividad parcial de los factores. Por el contrario, en la presente investigación se aborda la evaluación desde la perspectiva de la eficiencia con el método DEA, que es un enfoque relativamente novedoso en el país. La aplicación empírica se centra en un grupo de explotaciones agrarias con producción de frijol, en un municipio muy prometedor para este tipo de actividad como es San Genaro de Boconoíto, sector La Palaciera, ubicado en el estado Portuguesa, Venezuela. La identificación de las fincas eficientes, así como la medición de sus niveles de utilización de insumos relevantes, que constituyen estrategias diferenciales respecto a las fincas ineficientes, permitirá orientar las decisiones hacia la mejora de la capacidad competitiva de este último grupo de fincas. Este enfoque es relevante, ya que la gran mayoría de los estudios relacionados, especialmente en el estado Portuguesa (Alejua 2002), se han centrado en aspectos agroecológicos, técnicos, manejo de suelos, uso de productos químicos y de mejoramiento genético, entre otros.

Por otra parte, es importante elevar la producción nacional de alimentos dada la crisis de inseguridad alimentaria que enfrenta el país (González 2009), que se evidencia por el comportamiento histórico de las importaciones, ya que entre 1981 y 1990 la importación de alimentos y bebidas fue de 75,4 US\$ por persona y año. En la década siguiente (1991-2000) la cifra disminuyó a 65,9 US\$ por persona y año, y en la que acaba de concluir (2001-2010) se duplicó, alcanzando un promedio de 134,6 US\$ (Machado 2011).

Se escogió el método DEA por tres razones fundamentales: su mayor estandarización (con relación a otros métodos), por considerar simultáneamente múltiples insumos y productos y por su creciente utilización en el campo de las investigaciones agrícolas. Por otra parte, es relevante asociar variables de tipo socioeconómico y geoespacial con los resultados de eficiencia, en la búsqueda de factores explicativos, lo cual constituye el aporte más importante de la presente investigación. Por lo general, estos tres aspectos no suelen abordarse de manera integrada, tal como lo evidencian los estudios siguientes:

Gilillan (1998) aplicó el método DEA a una muestra de 409 fincas ubicadas en las regiones de Comayagua y Santa Bárbara, en Honduras, que explotaban varios cultivos, entre ellos el frijol. Concluyó que bajo la asunción de retornos variables a escala, las fincas más grandes son más eficientes tecnológicamente, pero más ineficientes a nivel de sus escalas de producción.

Perdomo et al. (2007) calcularon la eficiencia técnica y sus determinantes por tipo de caficultor (pequeño, mediano y grande) y sector general, para una muestra de 999 fincas, en los departamentos de Caldas, Quindío y Risaralda (Colombia). Como conclusión del estudio, se encontró, entre los resultados más destacados, que gran parte de los pequeños y medianos caficultores son ineficientes técnicamente, mientras que los grandes son eficientes en la práctica. Mediante el DEA, se obtuvo un puntaje 
promedio de eficiencia técnica de $36 \%$ en los pequeños, $51 \%$ en los medianos, $60 \%$ en los grandes y $42 \%$ en el sector general de caficultores de la zona central en estudio. Estos promedios de eficiencia técnica, comparados con estudios similares en África y Vietnam, indican una eficiencia técnica menor en los pequeños y grandes caficultores colombianos, confrontados con los mismos grupos en Vietnam, que también posee una vasta explotación agroindustrial de las plantas de café.

Perdomo y Mendieta (2007) recabaron datos microeconómicos de caficultores pequeños, medianos y grandes en los departamentos de Caldas, Quindío y Risaralda (Colombia), para determinar la eficiencia técnica y asignativa (o eficiencia precio) mediante el método no paramétrico Análisis Envolvente de Datos (DEA). La eficiencia técnica promedio encontrada para pequeños fue de $3,76 \%$, para los medianos de $51,71 \%$, para los grandes de $60,15 \%$ y todo el sector de $42,38 \%$. Los promedios de la eficiencia asignativa obtenida para los mismos grupos antes mencionados fueron de $36,13 \%, 42,98 \%$, $18,86 \%$ y $36,50 \%$, respectivamente.

$\mathrm{Al}$ extrapolar estas cifras los autores presuponen que el sector cafetero (independientemente del tamaño de la finca) presenta ineficiente técnica y asignativa, destacando que, de todos los grupos comparados, los grandes productores son los que presentan la mayor eficiencia técnica, pero también la más baja eficiencia asignativa. Esto significa que las unidades empresariales emplean muy bien la cantidad de insumos para maximizar su producción pero no logran producir al mínimo costo, dada sus ineficiencias asignativas. Por su parte, los minifundistas, los campesinos y el sector en general no explotan sus factores óptimamente y tampoco llegan al menor costo en producción por tener ineficiencia técnica y asignativa.

Por su parte, Ajibefun (2008) señaló que los análisis de la eficiencia son un tema importante en los estudios económicos. En una muestra de 200 fincas de pequeña escala de Nigeria, que incluían la explotación de frijol como cultivo asociado, comparó la capacidad de las técnicas paramétricas y no paramétricas de los modelos de frontera en los análisis de eficiencia técnica. Para este estudio, se empleó la técnica paramétrica conocida como modelo de Frontera de Producción Estocástica (FPE), y el Análisis Envolvente de Datos (DEA), para la técnica no paramétrica. Los resultados del análisis indican que los agricultores de la muestra tienen diferentes niveles de eficiencia técnica, que van desde 0,22 hasta 0,87 para ambas técnicas.

Según Mulwa et al. (2009) en las áreas de caña de azúcar al oeste de Kenia, hay presión sobre los agricultores para cultivar alimentos o explotar la caña de azúcar, que es el principal cultivo comercial. Además, debido a que se siembra en lotes pequeños de tierra, la productividad y la eficiencia son de gran importancia, tanto en cultivos comerciales como en los de subsistencia. En su trabajo utilizaron el DEA bietápico y la regresión de Tobit para evidenciar las deficiencias en el cultivo de maíz y sus causas, en el oeste de Kenia.

Koc et al. (2011) analizaron la eficiencia técnica de la segunda cosecha de maíz, durante el periodo 2004-2005, en 89 fincas localizadas en la región mediterránea oriental de Turquía. Aplicaron el método DEA y utilizaron la regresión Tobit para identificar los determinantes de esa eficiencia. Se empleó un modelo 
orientado a insumos, que permitió estimar que los productores podían reducirlos en por lo menos, un $19 \%$, manteniendo el mismo nivel de producción. Por otra parte, la eficiencia técnica estuvo en un rango entre 41 y $100 \%$.

En cuanto al empleo del DEA y los sistemas de información geográfico se tiene que Becerril et al. (2011) demostraron la existencia de patrones espaciales en los resultados de la eficiencia técnica del sector agropecuario en los estados mexicanos para el año 2008.

Samad et al. (2008) aplicaron el DEA para estimar la ecoeficiencia de empresas de algodón y elaborar un ranking de eficiencia técnica de las áreas algodoneras con base a una muestra de 53 fincas que cultivaban algodón bajo riego en la cuenca Mooki, situada en el norte de Nueva Gales del Sur. Estos resultados sirven para construir un sistema de clasificación que permita determinar la mejor orientación de las políticas, a fin de alcanzar los objetivos económicos y medioambientales.

\section{MATERIALES Y MÉTODOS}

\section{La medición de la eficiencia con el método DEA}

Por lo general, el análisis de la eficiencia en el sector agrícola se realiza a partir de indicadores técnicos parciales, tales como la producción por unidad de insumo utilizado: hectárea, jornal o capital. Estos indicadores sólo muestran una combinación particular de los recursos aplicados y del producto cosechado, por lo cual representan un enfoque que conlleva a una importante simplificación del proceso productivo agrícola, ya que no permite apreciar la producción como un resultado global, donde se concreta la suma e interacción de los factores que intervienen. Por tanto, se obvia la influencia en la obtención del producto de la proporción y forma de combinación de los distintos insumos aplicados. Así pues, un enfoque de la eficiencia agrícola basado en la relación global de productos e insumos resultará más apropiado para las fincas.

Un aporte importante fue el trabajo seminal de Farrell, en el año 1957, quien abordó la producción desde este enfoque global y estableció el marco teórico básico para estudiary medir la eficiencia (Farrell 1957). Esta teoría tiene gran aplicabilidad en el sector agropecuario, ya que el autor propuso el estudio de la eficiencia desde una perspectiva real y no ideal, donde cada finca puede sea evaluada con relación a otras que conforman una muestra representativa y homogénea de las fincas de una determinada región. Así, la medida de la eficiencia agrícola será relativa y no absoluta, donde el puntaje de eficiencia para una finca determinada refleja la desviación observada respecto a aquellas fincas consideradas como eficientes, que conforman la frontera de posibilidades de producción.

La teoría de Farrel (1957) se aplica en la práctica utilizando, principalmente, dos metodologías: las aproximaciones paramétricas y las no paramétricas. Para la primera se recurre al uso de la econometría y para la segunda se emplea el método DEA (por sus siglas en inglés Data Envelopment Analysis o Análisis Envolvente de Datos). Existen dos modelos básicos de DEA los cuales se describen a continuación.

\section{Modelo DEA-RCE}

Esta metodología fue originalmente desarrollada por Farrell (1957) y popularizada por Charnes et al. (1978). En ella se asume una frontera de producción con rendimientos constantes a 
escala (RCE), la cual se construye mediante la aplicación de métodos de programación lineal, de manera que el cálculo de la eficiencia de las fincas se realiza de los productos e insumos empleados en sus procesos productivos. De manera tal que un análisis de eficiencia con base a los insumos permite determinar la cantidad de insumos que puede ser proporcionalmente reducida manteniendo los niveles de productos que actualmente se están logrando en la finca. Por el contrario, el análisis enfocado en los productos permite determinar la cuantía en que puede incrementarse proporcionalmente el nivel de productos manteniendo fijas las cantidades de los insumos actualmente aplicados.

Una manera más intuitiva de abordar este modelo DEA es utilizando su expresión en forma de razones, pero comúnmente se emplea la versión dual del programa lineal, debido entre otras razones a que cada una de las variables que aparecen en él tienen un significado concreto y que es una formulación más sencilla, puesto que presenta un número menor de restricciones que sus primales (Martín 2005).

De manera formal, la solución de este modelo, con orientación a los productos, requiere resolver el problema de programación matemática siguiente:

$$
\begin{gathered}
\text { Minimizar } \theta_{0}-\varepsilon\left[\sum_{i=1}^{m} S_{i}^{+}+\sum_{r=1}^{s} S_{r}^{-}\right] \\
\text {s.a.: } \sum_{j=1}^{n} \lambda_{i} X_{i j}+S_{i}^{+}=\theta_{0} X_{i 0} \\
\sum_{j=1}^{n} \lambda_{j} Y_{r j}-S_{r}^{-}=Y_{r 0} \\
\lambda_{i}, S_{i}^{+}, S_{r}^{-} \geq 0 \\
j=1,2, \ldots \ldots ., n \\
i=1,2, \ldots \ldots, m \\
r=1,2, \ldots \ldots ., s
\end{gathered}
$$

En el caso del problema dual para el modelo $\mathrm{CCR}$, orientado al producto, $\mathrm{Xij}$ representa la cantidad de insumo i utilizado por la finca $j$.

La nomenclatura Yrj representa la cantidad de producto $r$ obtenido por la explotación j. Los valores $\lambda j$ son los pesos utilizados para calcular la ubicación de una finca ineficiente que se planea convertir en eficiente. De manera que las fincas ineficientes pueden ser proyectadas sobre la frontera de posibilidades de producción como una combinación lineal empleando estos pesos.

Por su parte, $\mathrm{S}_{i}^{+}$y $\mathrm{S}_{\mathrm{r}}^{-}$representan las variables de holgura de cada una de las $\mathrm{m}+\mathrm{s}$ restricciones del modelo y $\theta_{0}$ es la ratio de eficiencia de la finca que se está evaluando, de manera que este puntaje de eficiencia corresponde a la distancia entre una finca y la frontera de posibilidades de producción, que representa la combinación lineal de las mejores fincas de una muestra evaluada. Dado que el escalar $\theta$ asume valores menores o iguales a uno y se tienen los dos casos siguientes:

a) $\theta=1$ : en cuyo caso la finca analizada está ubicada sobre la frontera de posibilidades de producción, por lo cual se considera que su proceso productivo es eficiente.

b) $\theta<1$ : la finca analizada se encuentra al interior de la frontera de posibilidades producción, por lo cual se considera que su proceso productivo es ineficiente.

Para obtener el resultado eficiente de utilización de productos (orientación al producto) se multiplica cada valor observado de los productos por el porcentaje de incremento debido a la ineficiencia y a este resultado, se le suma el valor de la variable de holgura. 


\section{Modelo DEA-RVE}

Este modelo se debe a Banker et al. (1984) y a diferencia del modelo anterior, asume una frontera de producción convexa, construida al incorporar al modelo la restricción de que todas las ponderaciones asignadas a cada finca sumen la unidad $\sum_{\mathrm{j}=1}^{\mathrm{n}} \lambda_{\mathrm{j}}=1$

La solución de este modelo, en su versión primal con orientación a los productos, requiere resolver el problema de programación matemática siguiente:

$$
\begin{gathered}
\text { Maximizar } \theta_{0}+\varepsilon\left[\sum_{i=1}^{m} S_{i}^{+}+\sum_{r=1}^{s} S_{r}^{-}\right] \\
\text {s. a.: } \sum_{j=1}^{n} \lambda_{i} X_{i j}+S_{i}^{+}=X_{i 0} \\
\sum_{j=1}^{s} \lambda_{j} Y_{r j}-S_{r}^{-}=\theta_{0} Y_{r 0} \\
\sum_{j=1}^{n} \lambda_{j}=1 \\
\lambda_{i}, S_{i}^{+}, S_{r}^{-} \geq 0
\end{gathered}
$$

\section{Datos}

Mediante cuestionarios se recabó información de 30 explotaciones donde se cultiva frijol, ubicadas en el sector La Palaciera (localizado al sureste de Boconoíto, estado Portuguesa, Venezuela), en una zona delimitada por las coordenadas 967.428 y 972.199 UTM Norte y 403.582 y 412.772 UTM Este.

Para el análisis de la eficiencia se consideró como producto u output los kilogramos de frijol (Frijol) cosechados por finca, y cinco insumos $\mathrm{o}$ inputs considerados relevantes en el proceso productivo correspondiente al año 2010: número de hectáreas (ha) sembradas por finca, así como los gastos totales en bolívares erogados por los conceptos de: preparación de tierra (laboreo), de semilla (semilla), control de malezas (cmalezas) y de cosecha (cosecha). La incorporación de gastos como insumos, que es frecuente en los análisis de eficiencia, implica que la eficiencia estimada no es eficiencia técnica en sentido estricto, porque incluye elementos de la eficiencia asignativa, relacionados los precios de los insumos (Castillo 2006). En virtud de que, tanto a nivel local como nacional, la demanda de frijol excede a la oferta se empleó un modelo DEA orientado a los productos, el cual fue resuelto con el programa informático Win4deap (Coelli 1996). Otros cálculos estadísticos fueron realizados con el programa informático SPSS (IBM 2011).

Para analizar los determinantes socioeconómicos de la eficiencia se midieron en una primera instancia las variables: Sexo, Educación (3 niveles), Trabajos fuera de la finca (Si/No), Absentismo (Si/No), Mano de obra familiar (Número), Mano de obra contratada (Número), Edad (Años) y Distancia $(\mathrm{km})$ de la finca a la carretera asfaltada principal, pero solo hubo heterogeneidad en las 4 últimas, que fueron utilizadas en la presente modelización. Se empleó la regresión Tobit por ser la más idónea para este tipo de análisis (Bravo et al. 2007; Mulwa et al. 2009; Javed et al. 2010; Koc et al. 2011), ya que las regresiones censuradas evitan las estimaciones asintóticamente sesgadas de mínimos cuadrados ordinarios. Esta regresión fue estimada con el software Stata (StataCorp 2009).

También se analizó la posible influencia de otros factores en la eficiencia, tales como los edafoclimáticos o cercanía a carretera asfaltada principal más cercana, mediante un estudio de la distribución espacial de los puntajes de ETG (clasificándolos según superaran o 
no la media) obtenidos por cada finca, cuyas coordenadas x e y representan, por lo general, un punto muy cercado a la entrada del predio. Estas coordenadas fueron tomadas con un GPS marca Garmin serie GPSMAP 76CSX. Para el procesamiento geoespacial se aplicó el paquete informático ArcGis versión 9.2 (ESRI Inc. 2008). Se emplearon coordenadas UTM, Datum SIRGAS-REGVEN (WGS-84), zona 19N. También se utilizó Google Earth (Google Inc.) como herramienta de apoyo para desplegar la información generada.

\section{RESULTADOS Y DISCUSIÓN}

\section{Estimación de la eficiencia de las fincas productoras de frijol.}

Las 30 explotaciones de frijol objeto de estudio arrojaron una media de $850,7 \mathrm{~kg} / \mathrm{ha}$, valor inferior $(p>0.01)$ al promedio nacional calculado en $1.200,0 \mathrm{~kg} / \mathrm{ha}$ (Fedeagro 2012) y su desviación típica fue de $83,7 \mathrm{~kg} / \mathrm{ha}$.

Previo a la determinación de la eficiencia con el método DEA se realizó un análisis de correlación (Rho de Spearman) entre las variables del modelo DEA. Debido a que se dio una correlación perfecta entre ha y laboreo, se optó por eliminar este último insumo del análisis, tal como lo recomiendan diversos autores (Chediak 2008, pp. 90, 91; Chediak y Rodríguez 2011).

Las fincas estudiadas arrojaron una eficiencia técnica global (ETG) media de 81,2\% (Tabla 1), lo cual indica que sus producciones de frijol podrían incrementarse, en promedio en $23,2 \%$ ((1/0,812)-1); sin aumentar los recursos aplicados actualmente y operando al tamaño de escala más productivo. Por otra parte, el valor mínimo (ETG $=71,4 \%$ ) revela que la finca menos eficiente debería incrementar su producción en 40,1\% para alcanzar el nivel productivo de las 2 fincas eficientes del grupo comparado, que representan el $6,7 \%$ de la muestra.

El índice medio de eficiencia técnica pura $(\mathrm{ETP}=86 \%)$ permite estimar que la producción de las fincas ineficientes debería ser incrementada en $16,3 \%$, en promedio, para ser eficientes a la escala establecida por el grupo de 7 fincas con $100 \%$ de ETP, que representan

Tabla 1. Tres tipos de eficiencia de 30 explotaciones de frijol ubicadas en el municipio San Genaro del estado Portuguesa

\begin{tabular}{lccr}
\hline \multicolumn{1}{c}{ Concepto } & ETG (\%) & ETP (\%) & EE (\%) \\
\hline Mínimo & 71,4 & 74,3 & 75,2 \\
Máximo & 100,0 & 100,0 & 100,0 \\
Media & 81,2 & 86,0 & 95,1 \\
Mediana & 76,2 & 84,8 & 100,0 \\
Desviación típica & 8,2 & 10,4 & 8,7 \\
No de fincas eficientes & 2 & 7 & 19 \\
\% fincas eficientes & 6,7 & 23,3 & \\
\hline & & $10(33,3 \%)$ & \\
Número de fincas irs & & $1(3,3 \%)$ & \\
Número de fincas drs & &
\end{tabular}

irs: rendimientos a escala crecientes drs: rendimientos a escala decrecientes.

$\mathbf{E T G}=$ eficiencia técnica global $\quad \mathbf{E T P}=$ eficiencia técnica pura $\mathbf{E E}=$ eficiencia de escala 
el $23,3 \%$ de la muestra.

El índice de eficiencia de escala promedio $(E E=95,1 \%)$ refleja que hay ineficiencias debidas a que el $36,7 \%(100 \%-63,3 \%)$ de las fincas no están operando, en promedio, a sus tamaños óptimos (medido por los volúmenes de producto). Estas ineficiencias de escala se atribuyen menos a tamaños por encima del óptimo (1 finca que representa el 3,3\%) que a tamaños por debajo del óptimo, que presentan 10 fincas (33,3\% de la muestra) que operan con rendimientos a escala crecientes (irs). Estas fincas, que deberían aumentar su tamaño para ser más eficientes, conforman un grupo considerado como un problema estructural de la agricultura de algunos países (Papageorgiou y Spathis 2000).

Es importante destacar que la ineficiencia generada por el uso de la tecnología de producción es mayor en comparación con la ineficiencia de escala, lo cual justifica la formulación y ejecución de un plan de transferencia de tecnología, aunque la brecha tecnológica, representada en su límite inferior por el valor de la ETP mínimo, no es tan acentuado como el reportado en estudios de otros cultivos (Ajibefun 2008; Koc et al. 2011).
Según los resultados del contraste $U$ de Mann-Whitney la tecnología de las fincas con producción de frijol se ajusta a rendimientos constantes a escala $(p>0.05)$.

\section{Análisis de segundo nivel}

Debido a que los rendimientos constantes a escala resultaron relevantes y solo hay dos fincas con $100 \%$ de eficiencia, se categorizó el índice ETG siguiendo a Castillo (2006), quien lo aplicó en ganadería bovina y tomo como punto de corte un valor muy cercano a la media de ETG. En la presente investigación se empleó la media de la ETG para formar dos categorías: 1) Nivel de eficiencia inferior a la media (ETG $\leq 81,2 \%$ ) y 2 ) Nivel de eficiencia superior a la media (ETG>81,2\%), tal como se muestra en el tabla 2. Cada uno de los insumos y productos fueron medidos en totales por finca $y$, luego, promediados por grupo. Los resultados indican que no existen diferencias ( $p>0.05)$ en las cantidades de insumos aplicados en los procesos productivos en las fincas, discriminadas por niveles de eficiencia (Tabla 2), pero si hubo diferencias $(p<0.05)$ en la producción de frijol, lo cual explica las disparidades en los niveles de eficiencia de los dos grupos de fincas comparados.

Tabla 2. Comparación de valores promedios de insumos y producto para 30 explotaciones de frijol con 2 niveles de eficiencia de ETG

\begin{tabular}{|c|c|c|c|c|}
\hline \multirow[b]{2}{*}{ Variables } & \multirow{2}{*}{$\begin{array}{c}\text { Media } \\
\text { de la } \\
\text { muestra }\end{array}$} & \multicolumn{2}{|c|}{ Niveles de ETG } & \multirow[b]{2}{*}{$\begin{array}{c}\text { Mann- } \\
\text { Whitney (p) }\end{array}$} \\
\hline & & $\begin{array}{l}\text { Inferior a } \\
\text { la Media }\end{array}$ & $\begin{array}{l}\text { Superior a } \\
\text { la Media }\end{array}$ & \\
\hline Frijol (kg) & $7.832,3$ & $7.377,4$ & $8.618,2$ & $0,00^{* *}$ \\
\hline ha & 9,2 & 9,3 & 9,1 & $0,77 \mathrm{~ns}$ \\
\hline Semilla (Bs) & $2.520,0$ & $2.542,1$ & $2.481,8$ & $0,67 \mathrm{~ns}$ \\
\hline Cmalezas (Bs) & $3.220,0$ & $3.247,4$ & $3.172,7$ & $0,93 \mathrm{~ns}$ \\
\hline Cosecha (Bs) & $1.380,0$ & $1.378,9$ & $1.381,8$ & $0,61 \mathrm{~ns}$ \\
\hline
\end{tabular}

* Nivel de significación < 1\% ns: no significativo p: probabilidad 
TEMAS AGRARIOS - Vol. 18:(2) Julio -Diciembre 2013 (67 - 82)

Tabla 3. Comparación de valores promedios de productividad por hectárea de 30 explotaciones de frijol con dos niveles de eficiencia de ETG

\begin{tabular}{lrrr}
\hline \multicolumn{1}{c}{ Variables } & Media & \multicolumn{2}{c}{ Niveles de ETG } \\
de la & Suestra & Superior a & \multicolumn{2}{c}{$\begin{array}{c}\text { Superior a } \\
\text { la Media }\end{array}$} & la Media \\
\hline Kg/ha & 850,7 & 796,8 & 943,6 \\
Semilla (Bs/ha) & 274,0 & 274,7 & 272,7 \\
Cmalezas (Bs/ha) & 350,0 & 350,5 & 349,1 \\
Cosecha (Bs/ha) & 150,7 & 149,5 & 152,7 \\
\hline
\end{tabular}

Los datos de insumos y productos de cada nivel de eficiencia del cuadro anterior fueron divididos entre la superficie, a fin de evaluar el sistema agrícola en términos de intensidad de la producción (productividad) para cada grupo clasificado por nivel de eficiencia (Tabla 3). El grupo con un nivel de eficiencia inferior a la media erogó montos por hectárea ligeramente superiores por conceptos de semilla y control de malezas, pero inferiores en la partida cosecha. Por otra parte, presentó una menor productividad del frijol. Es importante destacar que la productividad media del frijol de la muestra de productores $(850,7 \mathrm{~kg} / \mathrm{ha})$ estuvo por debajo $(p<0.01)$ de la media nacional (1.200 kg/ha de frijol) estimada para el año 2011 (Fedeagro 2012).

En la tabla 4 se presenta un resumen de la información más relevante para el plan basado en el enfoque benchmarking, a fin de transferir tecnología de las fincas eficientes a las menos eficientes. Dicho plan debe ser acompañado de un informe detallado del "cómo" se realizan las mejores prácticas en las fincas eficientes, a fin de ejecutar de manera exitosa el trasvase de la tecnología.

Debido a que este plan tiene una orientación al producto y considera rendimientos variables a escala, las metas de insumos se refieren a las cantidades que se registraron en el proceso productivo evaluado y que deben ser aplicadas en el próximo ciclo productivo, con la salvedad de que en algunas fincas que presentaron holguras de insumos, sus metas incluyen disminuciones de los mismos. Por otra parte, en las fincas que presentaron 100\% de eficiencia técnica global (ETG) se mantienen intactos los niveles de insumos y productos registrados en sus procesos productivos, a diferencia de las fincas ineficientes, donde deberán incrementarse las producciones de frijol, para ser transformadas en agroempresas eficientes.

En síntesis, para el conjunto de fincas evaluadas, se puede elevar la producción agregada de frijol de $234.970 \mathrm{~kg}$ a $289.200 \mathrm{~kg}$, lo que supone un incremento de $54.230 \mathrm{~kg}(23,1 \%)$. Los incrementos no son tan importantes debido a que las fincas tienden a ser relativamente homogéneas en cuanto al uso de tecnología, debido en gran medida a que reciben asesoría técnica de parte de instituciones públicas.

La consecución de las metas está condicionada a la capacidad gerencial del equipo de trabajo responsable del plan de transferencia de tecnología y de los productores agrarios y obviamente, por los factores que inciden en la producción del cultivo, que pueden 
ser sociales, económicos, edafoclimáticos y genéticos, entre otros.

Determinantes socioeconómicos de la eficiencia en el cultivo de fríjol.

A fin de evaluar los efectos de cuatro factores socioeconómicos en la eficiencia en el cultivo de fríjol se aplicó un modelo de regresión Tobit, cuyos resultado indican que solamente la variable mano de obra familiar tuvo incidencia $(p<0.05)$ en la eficiencia de las fincas productoras de fríjol (Tabla 5). Del valor del coeficiente de esta variable (que tiene signo positivo) se infiere que por cada trabajador clasificado como mano de obra familiar en las fincas evaluadas, se reportó un incremento de $5,125 \%$ de eficiencia técnica global (ETG).

Tabla 4. Eficiencia, nivel actual de insumos y productos, fincas líderes y metas de producción de 30 explotaciones de frijol

\begin{tabular}{|c|c|c|c|c|c|c|c|c|c|c|c|c|}
\hline \multirow[b]{2}{*}{ Finca } & \multicolumn{4}{|c|}{ Tipo de eficiencia (\%) } & \multirow[b]{2}{*}{ ha } & \multicolumn{3}{|c|}{$\begin{array}{c}\text { Metas de } \\
\text { insumos(Bs) }\end{array}$} & \multirow{2}{*}{$\begin{array}{c}\text { Producción } \\
\text { actual } \\
\text { Frijol (kg) }\end{array}$} & \multicolumn{2}{|c|}{$\begin{array}{l}\text { Metas de } \\
\text { producción }\end{array}$} & \multirow[t]{2}{*}{$\begin{array}{l}\text { № Fincas } \\
\text { referenciadas }\end{array}$} \\
\hline & ETG & ETP & $\mathrm{EE}$ & $\begin{array}{l}\text { Tipo } \\
\text { Rend. }\end{array}$ & & semilla & Cmaleza & cosecha & & $\begin{array}{c}\text { Frijol } \\
\text { (kg) }\end{array}$ & $\begin{array}{c}\text { Frijol } \\
(\%)\end{array}$ & \\
\hline 1 & 98,0 & 98,5 & 99,6 & irs & 10 & 2,623 & 3,4 & 1,36 & 10 & 10,2 & 2 & 0 \\
\hline 2 & 76,2 & 79,0 & 96,4 & irs & 8 & 2,16 & 2,8 & 1,12 & 6,4 & 8,4 & 24 & 0 \\
\hline 3 & 88,2 & 88,6 & 99,6 & irs & 10 & 2,623 & 3,4 & 1,36 & 9 & 10,2 & 12 & 0 \\
\hline 4 & 100,0 & 100,0 & 100,0 & - & 10 & 2,7 & 3,5 & 1,4 & 10,5 & 10,5 & 0 & 0 \\
\hline 5 & 81,0 & 100,0 & 81,0 & irs & 5 & 1,35 & 1,75 & 700 & 4,25 & 5,25 & 19 & 0 \\
\hline 6 & 85,7 & 100,0 & 85,7 & irs & 5 & 1,35 & 1,75 & 700 & 4,5 & 5,25 & 14 & 0 \\
\hline 7 & 76,2 & 76,2 & 100,0 & - & 10 & 2,7 & 3,5 & 1,4 & 8 & 10,5 & 24 & 0 \\
\hline 8 & 84,8 & 84,8 & 100,0 & - & 10 & 2,7 & 3,5 & 1,4 & 8,9 & 10,5 & 15 & 0 \\
\hline 9 & 75,2 & 100,0 & 75,2 & drs & 20 & 5,4 & 7 & 2,8 & 15,8 & 21 & 25 & 0 \\
\hline 10 & 100,0 & 100,0 & 100,0 & - & 10 & 2,7 & 3,5 & 1,4 & 10,5 & 10,5 & 0 & 29 \\
\hline 11 & 76,2 & 76,2 & 100,0 & - & 10 & 2,7 & 3,5 & 1,4 & 8 & 10,5 & 24 & 0 \\
\hline 12 & 81,0 & 81,0 & 100,0 & - & 10 & 2,7 & 3,5 & 1,4 & 8,5 & 10,5 & 19 & 0 \\
\hline 13 & 71,4 & 88,2 & 81,0 & irs & 5 & 1,35 & 1,75 & 700 & 3,75 & 5,25 & 29 & 0 \\
\hline 14 & 76,2 & 76,2 & 100,0 & - & 10 & 2,7 & 3,5 & 1,4 & 8 & 10,5 & 24 & 0 \\
\hline 15 & 84,8 & 84,8 & 100,0 & - & 10 & 2,7 & 3,5 & 1,4 & 8,9 & 10,5 & 15 & 0 \\
\hline 16 & 75,2 & 75,2 & 100,0 & - & 10 & 2,7 & 3,5 & 1,4 & 7,9 & 10,5 & 25 & 0 \\
\hline 17 & 76,2 & 100,0 & 76,2 & irs & 5 & 1,35 & 1,75 & 700 & 4 & 5,25 & 24 & 0 \\
\hline 18 & 85,7 & 100,0 & 85,7 & irs & 5 & 1,35 & 1,75 & 700 & 4,5 & 5,25 & 14 & 0 \\
\hline 19 & 95,2 & 95,2 & 100,0 & - & 10 & 2,7 & 3,5 & 1,4 & 10 & 10,5 & 5 & 0 \\
\hline 20 & 75,2 & 78,0 & 96,4 & irs & 8 & 2,16 & 2,8 & 1,12 & 6,32 & 8,4 & 25 & 0 \\
\hline 21 & 74,3 & 74,3 & 100,0 & - & 10 & 2,7 & 3,5 & 1,4 & 7,8 & 10,5 & 26 & 0 \\
\hline 22 & 75,2 & 75,2 & 100,0 & - & 10 & 2,7 & 3,5 & 1,4 & 7,9 & 10,5 & 25 & 0 \\
\hline 23 & 74,3 & 74,3 & 100,0 & - & 10 & 2,7 & 3,5 & 1,4 & 7,8 & 10,5 & 26 & 0 \\
\hline 24 & 85,7 & 85,7 & 100,0 & - & 10 & 2,7 & 3,5 & 1,4 & 9 & 10,5 & 14 & 0 \\
\hline 25 & 75,2 & 98,7 & 76,2 & irs & 5 & 1,35 & 1,75 & 700 & 3,95 & 5,25 & 25 & 0 \\
\hline 26 & 76,2 & 76,2 & 100,0 & - & 10 & 2,7 & 3,5 & 1,4 & 8 & 10,5 & 24 & 0 \\
\hline 27 & 76,2 & 76,2 & 100,0 & - & 10 & 2,7 & 3,5 & 1,4 & 8 & 10,5 & 24 & 0 \\
\hline 28 & 85,7 & 85,7 & 100,0 & - & 10 & 2,7 & 3,5 & 1,4 & 9 & 10,5 & 14 & 0 \\
\hline 29 & 74,3 & 74,3 & 100,0 & - & 10 & 2,7 & 3,5 & 1,4 & 7,8 & 10,5 & 26 & 0 \\
\hline 30 & 76,2 & 76,2 & 100,0 & - & 10 & 2,7 & 3,5 & 1,4 & 8 & 10,5 & 24 & 0 \\
\hline Media & 81,2 & 86,0 & 95,1 & & 9 & 2,479 & 3,213 & 1,285 & 7,832 & 9,64 & 19 & \\
\hline Total & & & & & 276 & 74,366 & 96,4 & 38,56 & 234,97 & 289,2 & & \\
\hline
\end{tabular}

Tipo Rend: tipo de rendimiento irs: rendimientos a escala crecientes drs: rendimientos a escala decrecientes $\mathrm{ETG}=$ eficiencia técnica global 
Tabla 5. Modelo de regresión Tobit para variables socioeconómicas de 30 productores de fríjol

\begin{tabular}{|c|c|c|c|c|c|c|}
\hline Tobit regression & & & & & er of ob & 30 \\
\hline Log likelihood= & & & & & $2(4)=$ & 4,41 \\
\hline & & & & & chi $2=$ & 0,35 \\
\hline ETGFrijol & Coef. & Std. Err. & $\mathrm{t}$ & $P>|t|$ & $\begin{array}{r}{[95 \%} \\
\text { Int }\end{array}$ & $\begin{array}{l}\text { Conf. } \\
\text { all] }\end{array}$ \\
\hline Edad & $-0,04$ & 0,18 & $-0,20$ & 0,84 & $-0,40$ & 0,33 \\
\hline MOFamiliar & 5,13 & 2,41 & 2,12 & 0,04 & 0,16 & 10,09 \\
\hline MOContratada & $-1,66$ & 1,60 & $-1,03$ & 0,31 & $-4,96$ & 1,63 \\
\hline Distancia & 0,22 & 0,54 & 0,40 & 0,69 & 0,90 & 1,33 \\
\hline _cons & 82,64 & 13,21 & 6,26 & 0,00 & 55,49 & 109,79 \\
\hline /sigma & 8,11 & 1,14 & & & 5,76 & 10,46 \\
\hline
\end{tabular}

\begin{aligned} \hline Obs. summary & 1 left-censored observation at ETGFrijol $\leq 71,4 \\ & 27$ uncensored observations \\ 2 & right-censored observation at ETGFrijol $\geq 100\end{aligned}$

Aunque no fue estadísticamente significativa, se observa que la mano de obra contratada disminuye (su coeficiente tiene signo negativo) en la medida en que se dispone de mano de obra familiar, la cual parece ofrecer un mayor rendimiento o productividad laboral.

Sin embargo, la evaluación del modelo como un todo, indica que presenta problemas de validez. Así el test estadístico de máxima verosimilitud (-log likelihood ratio test) con una distribución Chi cuadrado de 4,41 y 4 grados de libertad, arrojó un $\mathrm{p}$ valor mayor de $0,05(p=$ de 0,35$)$. Por esta razón se aplicó la correlación Rho de Spearman, confirmando que ninguna de las variables socioeconómicas está relacionada $(p>0.05)$ con la eficiencia técnica global (ETG) en la muestra de productores de fríjol objeto de estudio. Incluso la variable mano de obra familiar (MO Familiar), que se comportó estadísticamente significativa en el modelo Tobit, aquí arrojó el coeficiente de correlación más alto $(0,321)$, pero su $p$ valor asociado fue mayor de $5 \%(8,3 \%)$, por lo cual se descarta como variable determinante de la eficiencia técnica y se recomienda continuar la contrastación empírica.

\section{Distribución espacial de los índices de ETG.}

La distribución espacial de los puntajes de ETG obtenidos por cada finca se muestran en la Figura 1, donde el valor 1 representa los que están ubicados por debajo de la media $(\mathrm{ETG}<81,2 \%)$ y el valor 2 a los que son iguales o superiores a la media. Estos valores se distribuyen muy aleatoriamente, por lo cual se infiere que no fueron influenciados por la distancia a carretera principal o por variables edafoclimáticas, debido quizás a que la asistencia técnica pública que recibieron posibilitó una mayor homogeneidad en los resultados de eficiencia.

\section{CONCLUSIONES}

En la muestra de explotaciones de frijol la eficiencia técnica global (ETG) fue de 81,2\%, desglosada en una eficiencia técnica pura (ETP) de $86,0 \%$ y una eficiencia de escala 


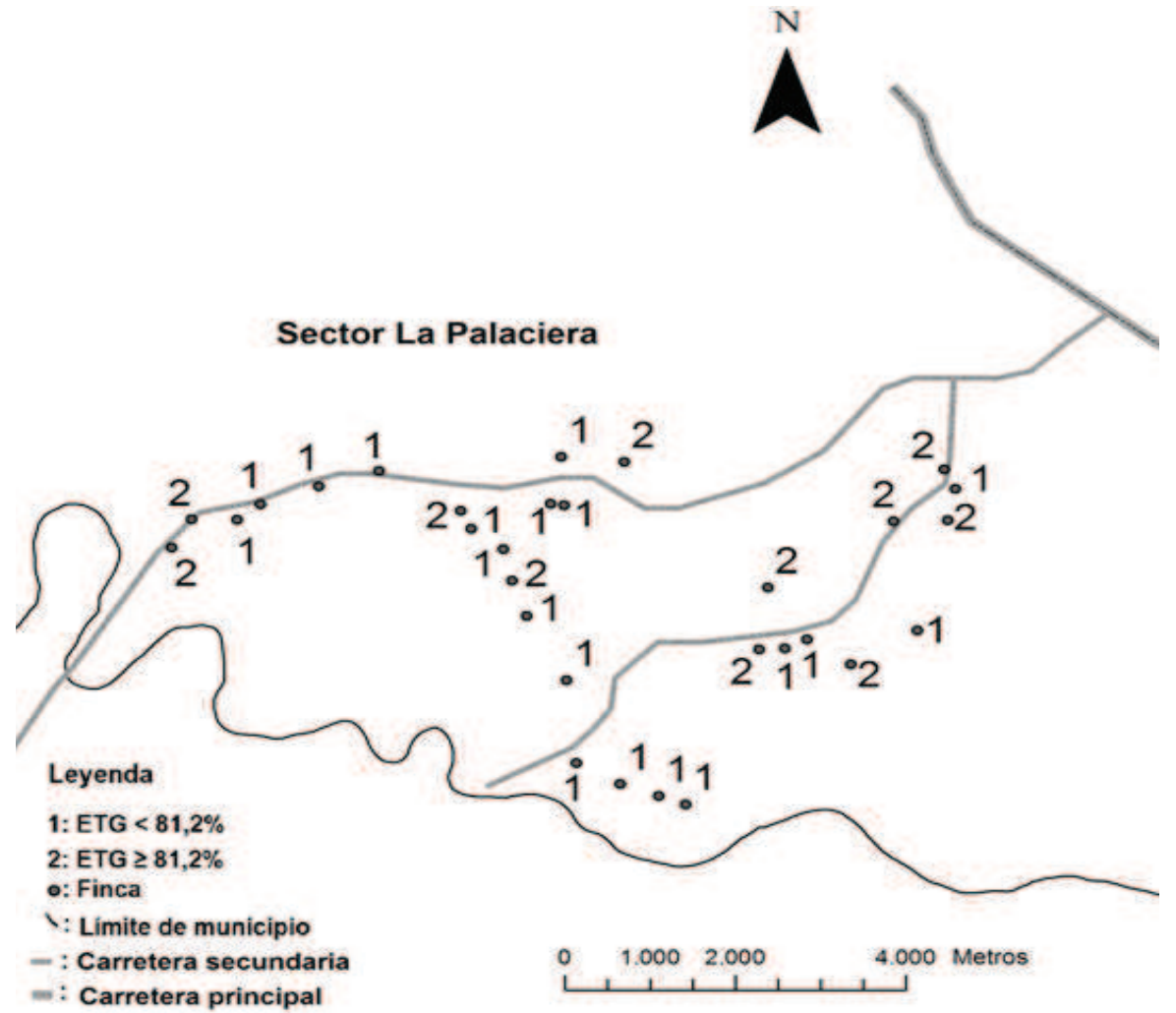

Figura 1. Distribución espacial de los puntajes de ETG obtenidos en 30 fincas productoras de frijol ubicadas en el municipio San Genaro de Boconoíto, sector La Palaciera del estado Portuguesa, Venezuela

Nota: El mapa de carreteras fue obtenido del laboratorio de Cartografía de la Universidad Ezequiel Zamora.

(EE) de 95,1\%. Asimismo, las ineficiencias causadas por la tecnología son mayores que las generadas por un tamaño o escala de producción subóptimo.

La tecnología de las fincas productoras de frijol se ajusta a rendimientos constantes a escala para el producto y los insumos analizados. En los estudios sobre el tipo de tecnología predominante en las explotaciones agrarias no se ha llegado a un consenso acerca de su ajuste a rendimientos constantes o variables a escala.

Según las metas del plan formulado para la muestra de explotaciones de frijol se puede elevar la producción agregada de $234.970 \mathrm{~kg}$ a $289.200 \mathrm{~kg}$, lo que supone un incremento de $54.230 \mathrm{~kg}(23,1 \%)$.

Debido a que los modelos están orientados a productos, estos incrementos se obtendrían con los niveles actuales de insumos (o con una pequeña reducción de los mismos, en algunos casos) aplicados a los procesos productivos, y no son significativamente más grandes porque las fincas tienden a ser relativamente homogéneas en cuanto al uso de tecnología debido, en gran medida, a que reciben asesoría técnica de parte de instituciones públicas.

De lograr concretarse estos aumentos de producción, se contribuiría a mejorar la 
seguridad agroalimentaria del país y también, a elevar la calidad de vida de los productores agropecuarios objeto de la planificación.

Las variables socioeconómicas y las relacionadas con factores del entorno como las edafoclimáticas o de cercanía a la carretera principal, asociadas a los sistemas de explotación del cultivo fríjol, no determinaron los niveles de eficiencia técnica de las fincas, ubicadas en el sector La Palaciera, municipio San Genaro del estado Portuguesa.

La causa más probable de que los hallazgos de esta investigación no concuerden con los reportados por Binam et al. (2003), Ríos y Shively (2005) y Koc et al. (2011), entre otros, es que la asistencia técnica pública que recibe el grupo de productores objeto de estudio, minimiza la incidencia de sus aspectos socioeconómicos y de variables del entorno en los niveles de eficiencia de sus explotaciones.

\section{REFERENCIAS}

Ajibefun, I. 2008. An Evaluation of Parametric and Non-Parametric Methods of Technical Efficiency Measurement: Application to Small Scale Food Crop Production in Nigeria. Journal of Agriculture \& Social Sciences 4: 95-100.

Alejua, H. 2002. Caracterización y análisis del proceso gerencial aplicado por los productores de maíz del municipio Turén, estado Portuguesa, Venezuela. Agroalimentaria 14:15-25.

Banker, R., Charnes, A. and Cooper, W. 1984. Some models for estimating technical and scale inefficiencies in data envelopment analysis. Management Science 30(9): 1078-1092.

\section{Becerril, O., Rodríguez G. y Ramírez J. 2011. Eficiencia técnica del sector agropecuario de México: Una perspectiva de análisis envolvente de datos. Economía, XXXVI (31): 85-110}

Binam, J., Silla, K., Diarra, I. and Nyambi, G. 2003. Efficient among coffee farmers in Côte d'Ivore: Evidence from the centre west region. R\&D Management, 15)1): 66-75.

Bravo, B., Solís D., Moreira V., Maripan J., Thiam A. and Rivas T. 2007. Technical efficiency in farming: a meta-regression analysis. J. Prod. Anal 27:57-72.

Castillo, M. 2006. Eficiencia técnica de la producción de vacuno de carne en la dehesa. Revista Española de Estudios Agrosociales y Pesqueros, $N^{\circ} 212: 139$ 154

Charnes, A., Cooper, W. and Rhodes, E. 1978. Measuring the efficiency of decision making units, European Journal of Operational Research 2(6):429-44.

Chediak, F. 2008. La Técnica DEA para medir y analizar la eficiencia municipios del departamento del Tolima, Año 2006. Tesis MSc.Universidad Tecnológica de Pereira. Facultad de Ingeniería Industrial, Pereira, Colombia.

Chediak, F. y Rodríguez Y. 2011. La eficiencia relativa en cobertura educativa 
de los municipios del Tolima, aplicando el análisis envolvente de datos -DEAAño 2009. ScientiaetTechnica 17(47): 44-48.

Coelli, T. 1996. A Guide to DEAP Version 2.1: A Data Envelopment Analysis (Computer) Program, CEPA Working Paper No. 8/96, University of New England, Department of Econometrics, England. 50 p.

ESRI Inc 2008. 380 New York Street, Redlands, CA 92373-8100, USA.

\section{Fedeagro (Confederación Nacional de Asociaciones de Productores Agropecuarios). 2012. Estadísticas agrícolas [Documento en línea]. http:// www.fedeagro.org/ produccion/default. asp. [junio de 2012].}

Farrel, M. 1957. The Measurement of Productive Efficiency. Journal of the Royal Statistical Society, Series A, 120, Part III: 253-290.

Flores, C., Madriz, P. y Warnock R. 2005. Evaluación de altura de plantas y componentes del rendimiento de seis genotipos del género Vigna en dos localidades de Venezuela. Rev. Fac. Agron., 22 (4): 354-368.

FUNICA (Fundación para el Desarrollo Tecnológico Agropecuario y Forestal de Nicaragua). 2011. Análisis de la cadena subsectorial del frijol. Papel de Trabajo. Nicaragua. $15 \mathrm{p}$.

Gilillan, D. 1998. Farm size, productivity and economic efficiency: accounting for differences in efficiency of farms by size in Honduras. University of Maryland. Agricultural and Resources Economics Departament, Mariland.15 p.

González, E. 2009. La inseguridad agroalimentaria de Venezuela. Tribuna del Investigador 10(1-2): 1-20.

Hernández, J. 2009. Evolución y resultados del sector agroalimentario en la $\mathrm{V}$ República. Cuadernos del Cendes $\mathrm{N}^{\circ} 72$ : 67-100.

IBM Corporation, 2011. Statistics 20. En: http://www.ibm.com/midmarket/ ve/es/?Ink=fti p\&cmp=Customer_ Journey_+_New_Client_Initiative\&ct $=1$ 02P531W\&cr $=$ Google \& $\mathrm{cm}=\mathrm{D} \& \mathrm{co}=\mathrm{On}$ $\& c c y=V E \& c s o t=W P: \& c p g=M i d M \& c p b=$ Other:\%20Other\&S_TACT=102P531W.

Javed M., Ali A., Adil S. and Raza, M. 2010. Measurement of technical efficiency of rice-wheat system in Punjab, Pakistan using DEA technique. J. Agric. Res. 48(2): 227-238.

Koc, B., Gul, M. and Parlakay, O. 2011. Determination of technical efficiency in second crop maize growing farmas in Turkey: case study for the east Mediterranean in Turkey. Asian Journal of Animal and Veterinary Advances 6 (5): 488-498.

Machado, A. 2011. Importación de alimentos en Venezuela ¿Qué sería razonable? [Documento en línea]. En http://carlosmachadoallison.blogspot. 
com/2011/02/ importacion-dealimentos-en-venezuela. html. [junio de 2012].

Marín, D. 2002. Rendimiento y producción agrícola vegetal: un análisis del entorno mundial (1997-1999) y de Venezuela (1988 - 2001). AgroalimentariaNº 15:4973.

Martín, R. 2005. La eficiencia en la asignación de recursos destinados a la educación superior: el caso de la Universidad de La Laguna. Tesis doctoral. Universidad de la Laguna. España. 231 p.

Mulwa, R., Emrouznejad, A. and Muhammad, L. 2009. Economic Efficiency of Smallholder Maize Producers in Western Kenya: A DEA Meta-Frontier Analysis. International Journal of Operational Research 4 (3): $250-267$

Papageorgiou, K. and Spathis, P. 2000. Agriculture Policy, Stochastis Editions, 1 st ed. Agriculture University of Athens, Athens, Greece.

Perdomo, J. y Mendieta, J. 2007. Factores que afectan la eficiencia técnica y asignativa en el sector cafetero colombiano: una aplicación con análisis envolvente de datos. Desarrollo y Sociedad N 60:1-45.
Perdomo, J., Hueth, D. y Mendieta, J. 2007. Factores que afectan la eficiencia técnica en el sector cafetero colombiano: una aplicación con análisis envolvente de datos. Ensayos sobre Economía Cafetera22:121-40.

Piccirillo, G y Higuera, A. 1997. Estudio de insectos polinizadores en el frijol, Vigna unguiculata (L.) Walp. y su efecto en el rendimiento. Rev. Fac. Agron. (LUZ) 14:307-314.

Ríos, A. and Shively, G. (2005). Farm size and nonparametric efficiency measurements for coffee farms in Vietnam. American Agriculture Economics Association. En: http://www.agecon.purdue.edu/staff/ shively/rs.pdf

Samad, M., Liem, M., Ancev, T. and Yu'Ting, L. 2008. Measuring Environmental Performance of Irrigated Cotton Enterprises. AARES 52nd Annual Conference Rydges Lakeside Canberra, ACT, Australia. 5-8 February 2008.

StataCorp. 2009. Stata Statistical Software: Release 11.1. College Station, TX: Stata Corporation. 